\title{
Improved Autolanding Controller for Aircraft Encountering Unknown Actuator Failures
}

\author{
Shaik Ismail, Abhay A. Pashilkar \\ Scientists, Flight Mechanics and Control Division \\ National Aerospace Laboratories \\ Bangalore - 560017, India \\ shaik1752@gmail.com, apash@nal.res.in
}

\author{
Ramakalyan Ayyagari \\ Prof., Dept. of Instrumentation and Control Engg., \\ National Institute of Technology \\ Tiruchirappalli, India \\ rkalyn@gmail.com
}

\author{
N. Sundararajan \\ Professor, School of Electrical \& Electronic Engineering, \\ Nanyang Technological University, Singapore 639798 \\ ensundara@ntu.edu.sg
}

\begin{abstract}
The authors have assessed the capability of various neural-aided classical feedback controllers that have been designed for autolanding of a typical modern high performance fighter aircraft under unknown actuator failures and external wind disturbances. Analysis of the fault tolerance envelopes of these neural-aided controllers revealed that position and rate saturation of the healthy actuators resulted in loss of control and failure to complete the autolanding task. Therefore, the over-all fault-tolerance region was not simply connected and exhibited gaps. In this paper we have successfully overcome the problem of gaps in the fault-tolerance envelope of the basic feedback controller by a judicious choice of feedback variables and developing a strategy for optimal gain selection to enlarge the failure tolerance envelopes in the presence of severe winds. The controller is motivated by Nonlinear Dynamic Inversion (NDI) approach and is able to handle six different types of single / double control surface failures. This is achieved by exploiting the full capability of control allocation inherent in the redundant control surfaces. The autolanding controller discussed in this paper is the most robust controller designed so far for the benchmark autolanding problem chosen for study.
\end{abstract}

Keywords-autolanding; non-linear dynamic inversion; control allocation; actuator failure;

\section{INTRODUCTION}

The developments in advanced technologies like the Instrument Landing Systems (ILS) and Global Navigation Satellite Systems (GNSS) have led to extensive use of autolanding for both the manned and unmanned aircraft. Autolanding ensures cost reduction, more safety and repeatability [1]. At present a majority of autolanding controllers are classical PID controllers which perform well under normal landing conditions but, fail under external disturbances like wind shear and turbulence, and aircraft component and actuator failure conditions [1]

Landing is the most critical flight phase of any air vehicle, and hence Fault Tolerant Control Systems (FTCS) for landing are being extensively investigated [1]. Neural networks with their ability to approximate nonlinear functions and capability of on-line learning provide a fast mechanism for adapting the aircraft control systems to unknown actuator failures, structural damage and wind disturbances, and hence are being increasingly used for fault tolerant flight control [2].

Using a six-degree-of-freedom (6 DOF) simulation model of a typical modern high performance aircraft with independent left and right elevator and aileron control surfaces [3], several neural-aided and neuro-fuzzy controllers have been reported in open literature for autolanding of the aircraft under unknown actuator failures and external wind disturbances [4-11]. The conventional PID, LQR, $H_{\infty}$ or $H_{2}$ controllers were used as baseline controllers for training the neural networks online. A glaring deficiency of these controllers is the presence of "holes" or gaps in the fault tolerance range or envelope of the controllers.

Efforts by the authors to fill the gaps in the fault tolerance regions of the neural-aided controllers that have been designed by them $[8,11]$ revealed that the rate and position saturations of the healthy actuators resulted in loss of control and failure to complete the autolanding task. This problem is more fully discussed in Section IV.

In this paper we have carefully looked at the design philosophy for the baseline controller with a view to address the gaps in the fault-tolerance region and also maximize the fault-tolerance envelope. In particular we paid attention to the following requirements:

- Multi-surface control allocation to increase the type of control surface failures being considered. This controller now handles six types of single / double control surface hard-over failures

- Optimal choice of gains for the inner and outer loops keeping in mind the contrary demands of ensuring adequate performance whist avoiding position/rate saturation of the actuators 
- Ability to handle severe wind conditions like microburst, cross winds etc.

The control structure was evolved from Nonlinear Dynamic Inversion (NDI) principles [13]. To the best of the knowledge of the authors, this is the most robust fault tolerant controller designed for the benchmark autolanding problem [8]. Enhancement of this controller with EMRAN based neural controller or Sliding Mode Controller did not improve the fault-tolerance capabilities. In some cases the neural-network was degrading the performance of the hybrid controller.

The rest of the paper is organized as follows: The autolanding problem is formulated in Section II. Section III also shows fault tolerance envelopes for a few neural-aided controllers that have been designed earlier and also introduces the NDI controller. The possible enhancements to this baseline controller are discussed in Section IV. The fault tolerance feasibility maps derived through 6 DOF simulations of the autolanding task are discussed in Section V. Finally, the conclusions drawn from the present study and suggestions for future work are given in Section VI.

\section{AUTOLANDING PROBLEM FoRMULATION}

\section{A. Aircraft Model}

The high performance fighter aircraft model chosen for the present study has conventional control surfaces, but with independent left and right elevator and aileron controls. CFD method was used to generate additional aerodynamic data for the split elevator and aileron control surfaces [3]. The two elevators can be moved together or in differential mode $(-25$ to $+25 \mathrm{deg})$. The deflection range for the independent ailerons is -20 to +20 deg., and for the rudder it is -30 to $+30 \mathrm{deg}$.

\section{B. Actuator Models and Failure Scenarios}

The hydraulic actuators are modeled as first order lags with a time constant of 50 millisec, and a rate limit of $60 \mathrm{deg} / \mathrm{s}$. In the present study six types of actuator failures are considered: failure of left elevator alone, failure of either left or right aileron alone, combined failure of left elevator and left aileron, combined failure of left elevator and right aileron, combined failure of both the ailerons, and failure of rudder alone. Failure of both the elevators is not considered because this case is, in general, not recoverable.

Failure of actuators can occur at any time during the flight. In the present study failures were injected just before the two critical stages of the landing flight: level turn and descent phases. Further, the failed control surfaces can be stuck at any value within the permissible range of deflections.

\section{Landing Trajectory and Wind Profiles}

The autolanding trajectory chosen for study is shown in Fig. 1. The trajectory consists of segments such as wings-level flight at $600 \mathrm{~m}$ altitude, two coordinated level turns, glide slope descent and finally the flare maneuver and touchdown on the runway. Two level turns are included in the trajectory so that the first turn segment can be used for on-line training of neural networks, and actuator failures are injected just before the second turn.
The wind disturbances are assumed to be present along all the axes throughout the landing mission, and are modeled on Dryden spectrum as shown in Fig. 2.

\section{Safety and Performance Criteria}

The desired touchdown point of aircraft under normal operating conditions is: $\mathrm{x}=0 \mathrm{~m}, \mathrm{y}=0 \mathrm{~m}$ and $\mathrm{z}=0 \mathrm{~m}$. Since the ideal touchdown cannot be achieved under unknown actuator failures, some safety and performance criteria need to be satisfied as specified and given below:

- $\mathrm{X}$-distance and Y-distance: $-100 \mathrm{~m} \leq \mathrm{x} \leq 400 \mathrm{~m}$, $-5 \mathrm{~m} \leq \mathrm{y} \leq 5 \mathrm{~m}$, to restrict the landing area to a rectangle of $500 \mathrm{~m} \times 10 \mathrm{~m}$, also called as "Pillbox".

- Total velocity: $V_{T} \geq 60 \mathrm{~m} / \mathrm{s}$ to prevent stall

- Sink rate: $\dot{h} \geq-2 \mathrm{~m} / \mathrm{s}$, to prevent landing gear damage

- Bank angle: $|\phi| \leq 10 \mathrm{deg}$, to prevent wing tips touching the ground

- Heading angle error: $|\psi| \leq 15 \mathrm{deg}$, to prevent excessive side loads on landing gear

\section{E. Fault Tolerance Feasibility Regions}

It is obvious that all the possible actuator stuck positions can not be accommodated by any controller because in some cases the resulting moments cannot be trimmed out for the landing maneuver, that is, a steady level turn or wings level descent may not be possible. Thus, the full range of hard over positions must be checked for the feasible subset.

The feasible range is computed by trimming the aircraft model with the control surfaces in failed positions. If trim is achieved then that particular failed position belongs to the feasible region. In the present work, the feasible region for autolanding is the union of the following trim computations:

- Region of level flight trim: body axis rates and flight path angle $\mathrm{p}=\mathrm{q}=\mathrm{r}=\gamma=0,6$ DOF accelerations $=0$

- Region of level descent trim: body axis rates $\mathrm{p}=\mathrm{q}=\mathrm{r}=0$, flight path angle $\gamma=-6 \mathrm{deg}, 6 \mathrm{DOF}$ accelerations $=0$

- Region of level turning trim: bank angle $\phi=40 \mathrm{deg}, 6$ DOF accelerations $=0$

\section{DYNAMIC INVERSION AUTOLANDING CONTROLLER DESIGN}

In [11], the authors have proposed to augment the basic trajectory following controller (BTFC) with a Neural Controller (EMRAN) as well as Sliding Mode Controller (SMC). Although the EMRAN augmentation distinctly improves the baseline controller performance for one elevator, and one aileron combination failure, we notice significant gaps in the failure tolerance regions of the controller (Fig. 3). The gaps can be filled by the use of a simple Phase Compensation (PC) scheme as shown in Fig. 4. In this paper we use a NDIbased feedback controller that has been designed by us [13] not only to expand the fault-tolerance envelopes, but also to avoid gaps in them. 


\section{A. Autolanding Controller Architecture}

A generic architecture for autolanding controllers is shown in Fig. 5. The controller comprises of two parts - a Tracking Command Generator that generates the commands based on trajectory deviations, and a Feedback Controller (FC) that accepts the command signals as its inputs. The FC is designed to make the closed-loop system stable along the desired trajectory, and to meet the performance requirements with or without actuator failures. As mentioned earlier, the FC can be realized by any approach like PID control, LQR, $H_{\infty}$ or $H_{2}$ control. In the present study the $\mathrm{FC}$ was developed using dynamic inversion concepts.

The Tracking Command Generator determines the offset of the aircraft from the desired ground track for each segment of flight which can be approximated by straight lines or arcs of circles. The reference commands consist of Altitude $\left(h_{r e f}\right)$, Velocity $\left(V_{\text {ref }}\right)$, Cross Distance from the desired track vector $\left(\delta_{r e f}\right)$, and the angular error of the aircraft velocity vector from the desired track vector $\left(\psi_{\text {ref }}\right)$.

\section{B. Full State Feedback Controller (FSFC)}

Nonlinear dynamic inversion with full state feedback is used to develop the baseline architecture called as Full State Feedback Controller (FSFC). The architecture of the baseline FSFC controller is shown in Figs. 6(i)-(ii). High gains are used in the inner loops, $K_{q}=-105 \mathrm{deg} / \mathrm{rad} / \mathrm{s}$ in the pitch rate loop, $K_{p s}=-25 \mathrm{deg} / \mathrm{rad} / \mathrm{s}$ in the stability axis roll rate loop, and $K_{r s}=-180 \mathrm{deg} / \mathrm{rad} / \mathrm{s}$ in the stability axis yaw rate loop to increase the robustness of the controller to actuator failures. The outer loop bandwidth is kept low to ensure that high frequency commands are not generated in response to disturbances due to severe winds.

The design begins by considering the three axes for feedback control. The equations of motion have been taken from [12]. The roll rate and yaw rate have been transformed into stability axes for the purpose of developing the control laws based on dynamic inversion. The details of controller design are given in [13]. Successful decoupling between the longitudinal and lateral-directional axes is achieved by the use of stability axis roll, pitch and yaw rates. The decoupling is further improved if the angle of attack used in the body to stability axis transformation is the 1 -g trim value

Multiple surface redundancy is used effectively to enhance the ability of the controller to handle failures like both ailerons failed. The robustness of the baseline FSFC controller to aileron and rudder failures is enhanced by using the independent elevator control surfaces in differential mode for the control of roll and yaw responses of the aircraft also, as shown below:

$$
\left[\begin{array}{l}
\delta_{\text {e-left }} \\
\delta_{\text {e-right }} \\
\delta_{\text {a-left }} \\
\delta_{\text {a-right }} \\
\delta_{\text {rudder }}
\end{array}\right]=\left[\begin{array}{ccc}
1.0 & -0.75 & -0.27 \\
1.0 & 0.75 & 0.27 \\
0 & -1.0 & 0 \\
0 & 1.0 & 0 \\
0 & 1.66 & 1.0
\end{array}\right]\left[\begin{array}{l}
\delta_{\text {pitch }} \\
\delta_{\text {roll }} \\
\delta_{\text {yaw }}
\end{array}\right]
$$

The gain entry of 1.66 is the aileron to rudder interconnect. The entries \pm 0.75 represent the use of elevators in differential mode to achieve control in the roll axis. This gain allows us to tolerate the additional failure case where both ailerons failed. Similarly, the gain entries \pm 0.27 are intended to create differential elevator control in response to demand for control in the yaw axis and enhance the ability of the FSFC to handle rudder failures.

A few comments are in order here. Firstly, the structure of this controller is based on non-linear dynamic inversion, where a few key stability and control derivatives are used, and other derivatives do not play a significant role. The cascade structure of the controller is designed with well separated time constants in the inner and outer loops. Finally, the design of the controller is easily extended to any other fixed wing aircraft, and in that sense it has universal application to this class of aircraft.

\section{IMPROVING FSFC AUTOLANDING CONTROLLER}

The principal aim of the autolanding controller is to reject winds and follow the predefined inertial trajectory for autolanding. Therefore, it seems appropriate to replace whenever possible the air-data feedback of angle of attack and sideslip with physically similar inertial signals. In the case of angle of attack, we have considered the pitch attitude for feedback. However, in case of the sideslip, placement of a low pass filter, with a first order time constant of $30 \mathrm{msec}$, in the feedback loop seemed to be the most appropriate solution.

Next, the failure cases for the FSFC were examined and it was found that the performance degrades due to the saturation of the rudder actuator due to the aileron to rudder gain. Therefore, the aileron to rudder interconnect gain was reduced to a value of 1.2 from its desired value of 1.66.

In fact, when a detailed study was conducted to identify the reasons for the "gaps' in the fault-tolerance envelope, it was found that these are mainly due to position/rate saturation of the actuators. Position saturation results in open loop response due to loss of regulatory action. On the other hand, rate saturation of the actuators causes a significant reduction in the phase bandwidth of an actuator when the amplitude of oscillation is increased. This is illustrated in Fig. 7. It can be seen that up to about $10 \%$ of the actuator amplitude, the frequency response of the actuator is close to the desired transfer function $(20 \mathrm{rad} / \mathrm{s}$ bandwidth), whereas at about $50 \%$ of the amplitude of oscillation, the effective bandwidth is about half of the desired value due to rate limiting in the actuator.

\section{A. EMRAN aided FC}

The basic principle of EMRAN aiding the FC has already been well developed in [8]. It is noted that this development is 
based on non-linear dynamic inversion concepts as explained in [2] and [8]. The inputs to the neural controller comprise of desired attitude rates $\left(\mathrm{p}_{\mathrm{d}}, \mathrm{q}_{\mathrm{d}}, \mathrm{r}_{\mathrm{d}}\right)$, the stability axis angular rates $\left(\mathrm{p}_{\mathrm{s}}, \mathrm{q}_{\mathrm{d}}, \mathrm{r}_{\mathrm{s}}\right)$, the angle of attack and side-slip angle $(\alpha, \beta)$ and control surface deflections $\left(\delta_{\mathrm{e}}, \delta_{\mathrm{a}}, \delta_{\mathrm{r}}\right)$. The desired rates are taken from the Feedback Controller (FC). The output comprises of neural controller outputs for the five aerodynamic control surfaces that tend to drive the differences between the desired and actual control surface signals to zero.

\section{B. SMC concept for FC}

In a neural-aided controller, the rate of learning of the neural network can be increased for faster recovery from failures. But, high rates of learning may excite unmodelled dynamics leading to stability problems. However, instead of increasing the rate of learning or choosing higher gains within the baseline controller, another controller which comes into play when large feedback errors are detected signaling failures can be used. The main task of this controller is to recover from failure using, if required, the full authority of the control surfaces which are healthy. In the present work the utility of a Sliding Mode Controller (SMC) is proposed for this purpose [11]. The overall scheme for neural and SMC aided Feedback Controller is shown in Fig. 8.

Classical SMC controllers were designed, for both the longitudinal and lateral-directional axes, using a simple sliding surface:

$$
\mathrm{S}=\widetilde{\mathrm{x}}+\lambda \int_{0}^{\mathrm{t}} \tilde{\mathrm{x}} \mathrm{d} \tau
$$

where, $\widetilde{x}=\mathrm{x}-\mathrm{x}_{\mathrm{d}}$ is the state tracking error and $\mathrm{x}_{\mathrm{d}}$ is the desired trajectory. The SMC in the longitudinal channel tracks the pitch rate, and the SMC in the lateral channel tracks the roll rate. The sliding mode control law is composed of two modes: $\mathrm{u}_{\mathrm{smc}}=\mathrm{u}_{\mathrm{eq}}+\mathrm{u}_{\mathrm{sat}}$, where $\mathrm{u}_{\mathrm{eq}}$ is the equivalent control signal and $\mathrm{u}_{\text {sat }}$ is the switching control signal. Simple PI block was used to compute the sliding surface $S$ which was then input to saturation blocks to obtain $\mathrm{u}_{\text {sat }}$.

\section{FAUlT TOLERANCE MAPS}

The first step in the evaluation of fault-tolerance capabilities of any autolanding controller, using six DOF simulations, is the examination of its feasibility regions for various types of actuator failures.

\section{A. Fault Tolerance Feasibility Maps}

The feasibility maps for the Feedback Controller are shown in Figs. 9-14 for various types of actuator failures. It is noted from these feasibility maps that the fault tolerance envelopes of the autolanding controller proposed in this paper (FC) are nearly the same as the corresponding fault tolerance envelopes of other controllers (FC+SMC+EMRAN) designed for the same autolanding problem in all cases of actuator failures except the case of (Left aileron + Right aileron) failure. In the latter case the optimally tuned controller (FC) performs better than the one augmented with EMRAN and SMC $(\mathrm{FC}+\mathrm{SMC}+\mathrm{EMRAN})$.

\section{CONCLUSIONS AND FUTURE WORK}

A robust linear flight controller with a cascade control structure is designed in reference [13] using the principle of NDI for generic fixed wing aircraft. The high-gain NDI controller is further improved in this paper to address the problem of auto landing for a high performance fighter aircraft, under severe winds and unknown actuator failures. The main findings are as follows:

- The gains in the innermost rotational equation loop should be chosen as high as possible to handle hardover control surface failures while at the same time low enough to prevent nonlinear actuator limits getting excited (position and rate limiting). Outer loop trajectory following gains are a compromise between a low value (to ensure that the flight controller does not create large inner loop demands and saturate the actuators when it encounters severe winds) and high value (to ensure that closed loop performance in terms of accurate trajectory following is achieved)

- The controller is able to handle severe winds by replacing the air-data sensors (e.g., angle of attack) by the feedback of an equivalent inertial signal (e.g., pitch attitude). Similarly, sharp changes in sideslip due to severe winds are prevented from affecting controller performance by introducing a low pass filter in the feedback path

- Multiple redundancies in control surfaces are handled by design of suitable interconnect gains. Where actuator rate limiting is a concern, exact control decoupling can be sacrificed to achieve better faulttolerance envelope

- Actuator failure tolerance range maps, also called as feasibility maps, show that the proposed controller is more robust than the other controllers reported in open literature for the same autolanding problem. Besides, the proposed controller can handle more types of actuator failures than the previous controllers (up to six).

- Previous results by the authors have shown that in cases where the Feedback Controller (FC) gains are lower than optimal, SMC and EMRAN based controllers are able to significantly improve the failuretolerance envelope $[8,11]$. In this study, we have established an optimal strategy for gain selection for the baseline controller and therefore we find that attempting to aid the FC with either EMRAN or SMC or both control architectures does not improve the fault tolerance significantly. In fact, in the case of double aileron failures degradation in performance is noticed. This is due to actuator rate limits which are excited by sharp transients from the sliding mode saturation 
component and the feed forward architecture of the EMRAN architecture.

\section{ACKNOWLEDGMENTS}

The authors express their sincere thanks to the organizations CSIR-NAL Bangalore, and NIT Tiruchirappalli for their support throughout the course of this work.

\section{REFERENCES}

[1] C. Edwards, T. Lombaerts, and H. Smaili, Fault Tolerant Flight Control: A Benchmark Challenge, $1^{\text {st }}$ ed, Springer-Verlag, New York, 2010.

[2] N. Sundararajan, P. Saratchandran, and Y. Li, Fully Tuned radial Basis Function Neural Networks for Flight Control, Boston:Kulwer Academic Publishers, 2001.

[3] S. G. Teo, Autolanding System Study: Aerodynamic Data on an Aircraft with Independent Control Surfaces by CFD, Technical Report of DSO National Lab., Singapore, 2003.

[4] W. Zhang, Control of a High Performance Aircraft by Using $H_{\infty}$ Theory and Neural-Fuzzy Concepts, M. Engg. Thesis, School of EEE, NTU Singapore, 1997.

[5] Y. Li, N. Sundararajan, and P. Sarathchandran, "Neuro-controller design for nonlinear fighter aircraft maneuver using fully tuned RBF networks," Automatica, vol. 37, pp. 1293-1301, 2001.

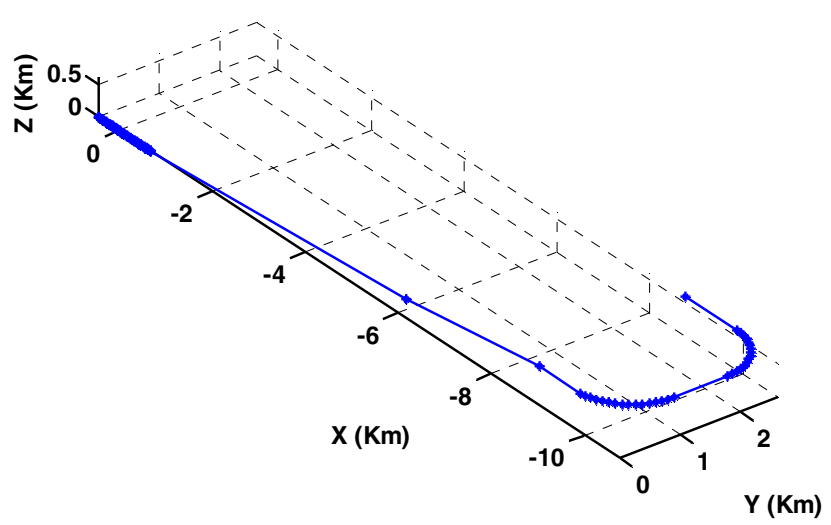

Fig. 1 Landing Trajectory

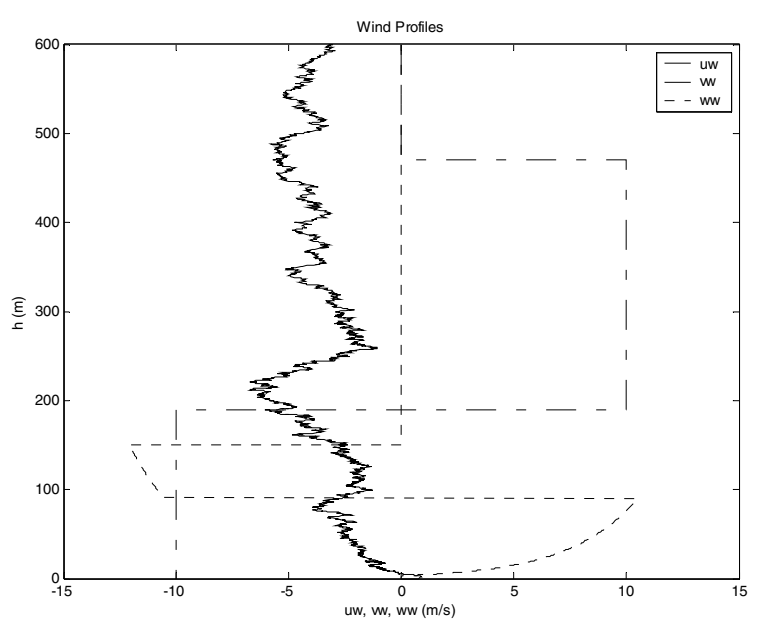

Fig. 2 Wind Profiles During Autolanding
[6] L. Fang, and W. Jianliang, Design of a Reliable Autolanding Control, Final Report, Project DSOCLO1144, School of EEE, NTU Singapore, 2004.

[7] Y. Li, N. Sundararajan, P. Saratchandran, and Z. Wang, " Robust neuro$H_{\infty}$ controller design for aircraft autolanding," IEEE Trans. on Aerospace and Electronic Systems, vol. 40, no. 1, pp.158-167, January 2004.

[8] A. A. Pashilkar, N. Sundararajan., and P. Saratchandran, "A faulttolerant neural aided controller for aircraft autolanding," Aerospace Science \& Technology, vol. 10, pp. 49-61, 2006.

[9] H. -J. Rong, N. Sundararajan, and P. Saratchandran, "Adaptive fuzzy fault-tolerant controller for aircraft autolanding under failures," IEEE Trans. on Aerospace and Electronic Systems, vol. 43, no. 4, pp.15861602, October 2007

[10] Z. Wang and G. Xiong, "Neuro-aided $\mathrm{H}_{2}$ controller design for aircraft under actuator failure," Proc. of the $20112^{\text {nd }}$ Intl. Congress on Computer Applications and Computational Science, vol. 2, 2011.

[11] S. Ismail, A. A. Pashilkar, and R. Ayyagari, "Guaranteed stability and improved performance against actuator failures using neural-aided sliding mode controller for autolanding task," Proc. IFAC-EGNAC 2012 Workshop, IISc Bangalore, 13-15 Feb. 2012.

[12] A. Miele, Flight Mechanics Volume 1: Theory of Flight Paths, AddisonWesley Publishing Company, Inc., 1962.

[13] A. A. Pashilkar, S. Ismail, R. Ayyagari and N. Sundararajan, "Design of nonlinear dynamic inversion controller for trajectory following and maneuvering for fixed wing aircraft", in Proc. CISDA 2013, Singapore, 16-19 April 2013.

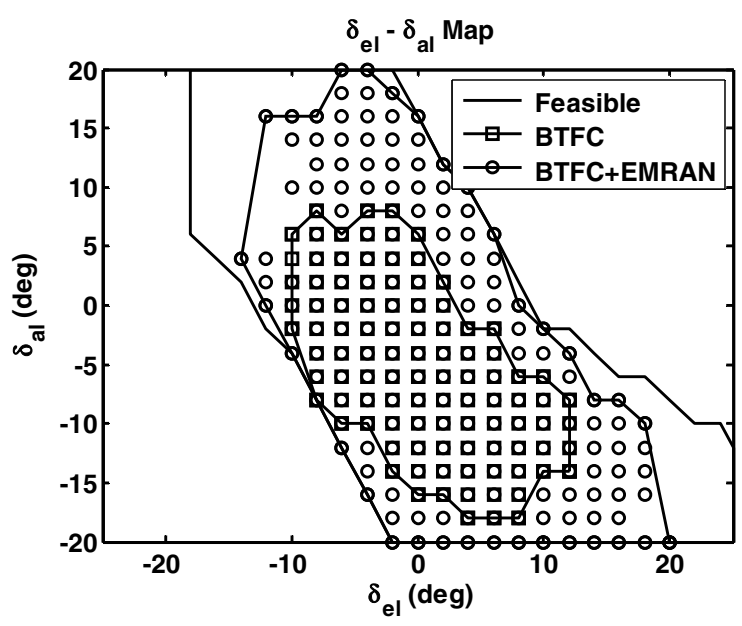

Fig. 3 Fault recovery from (left ele.+ left ail.) failures

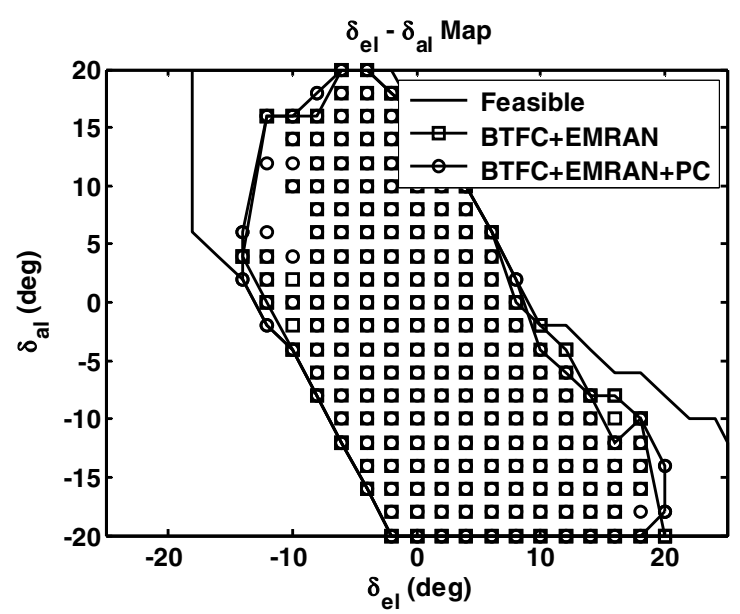

Fig 4. Fault recovery from (left ele.+ left ail.) failures with PC 


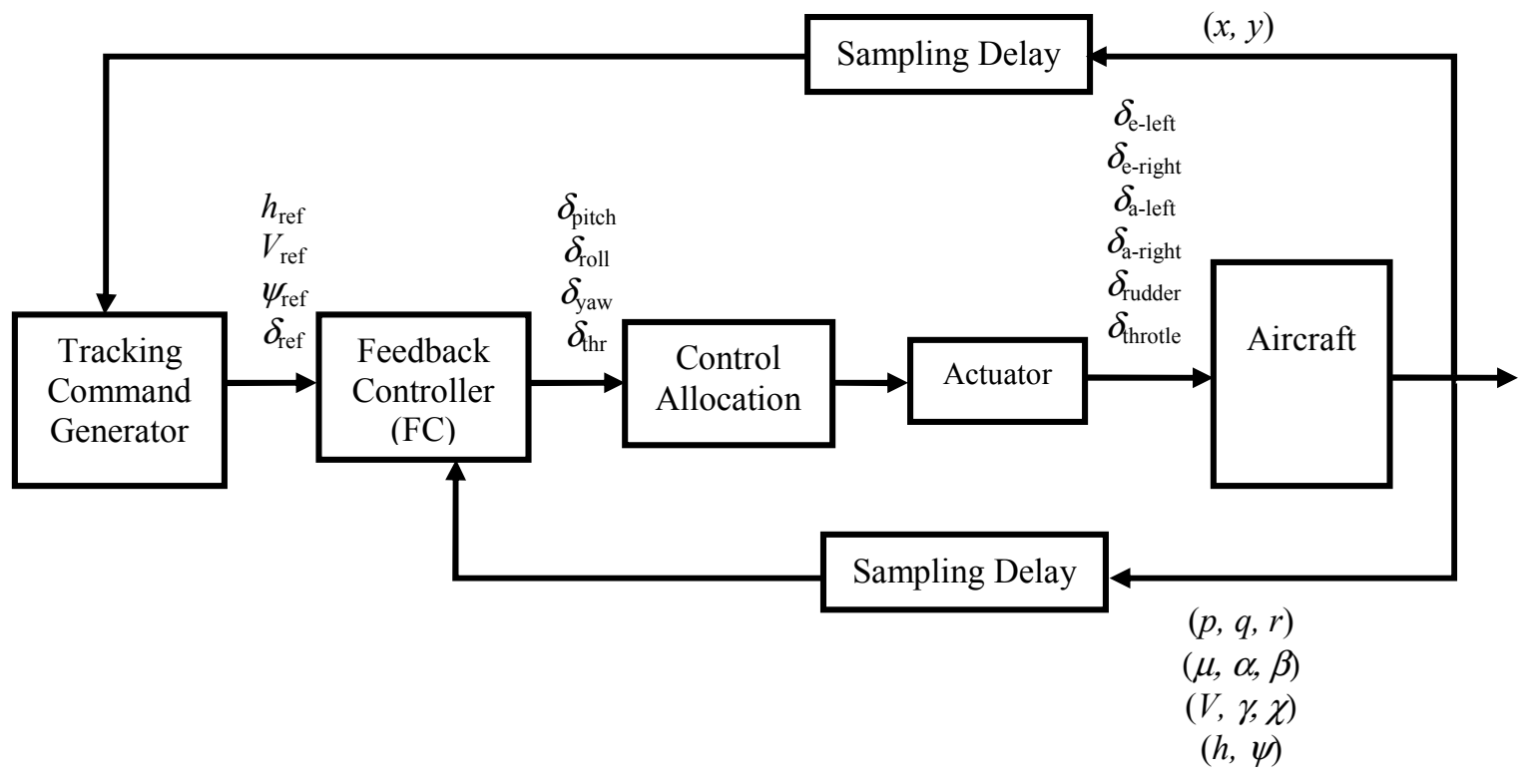

Fig. 5 Schematic of Flight Controller

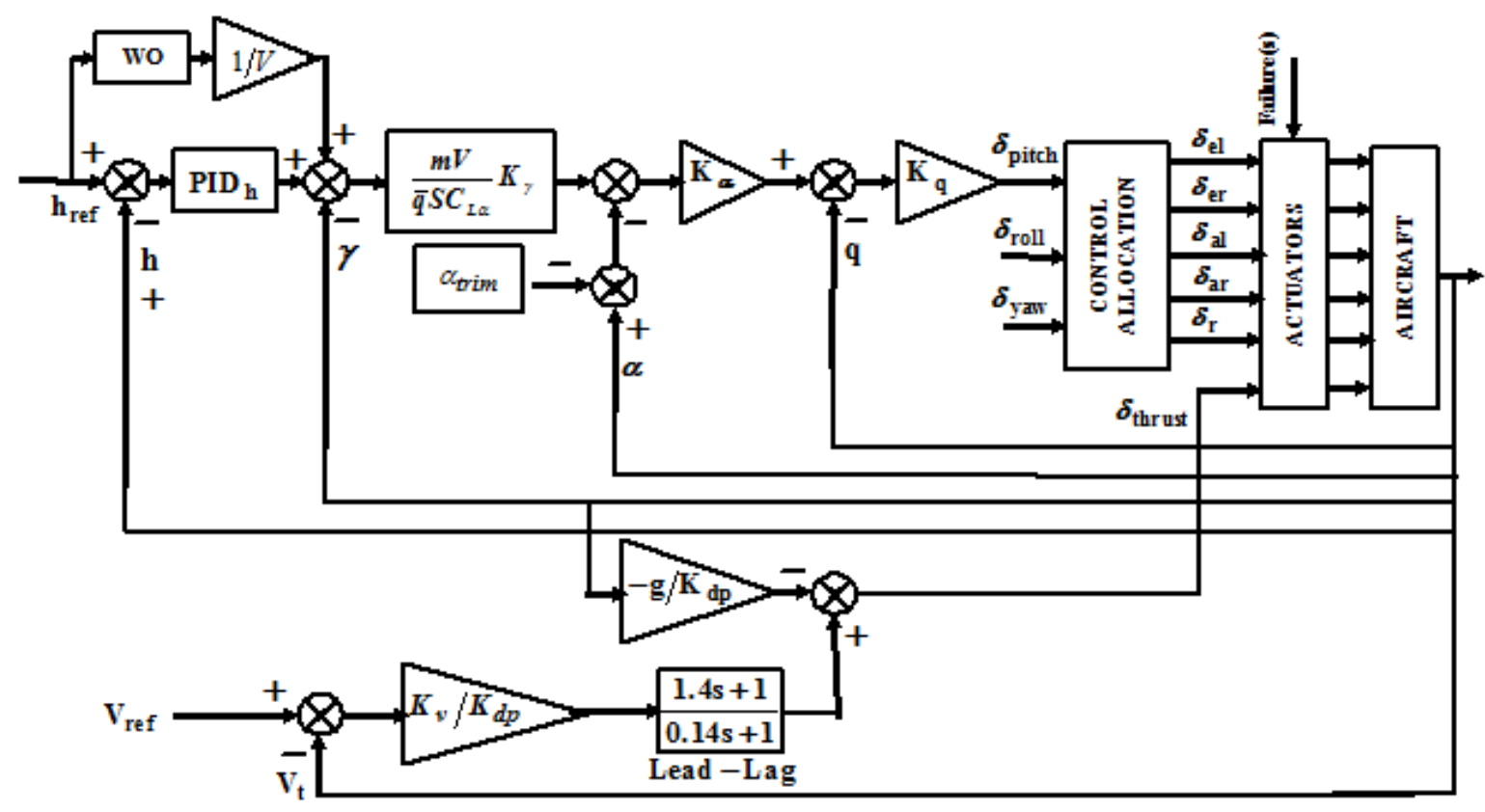

Fig. 6(i) Longitudinal Axis FSFC 


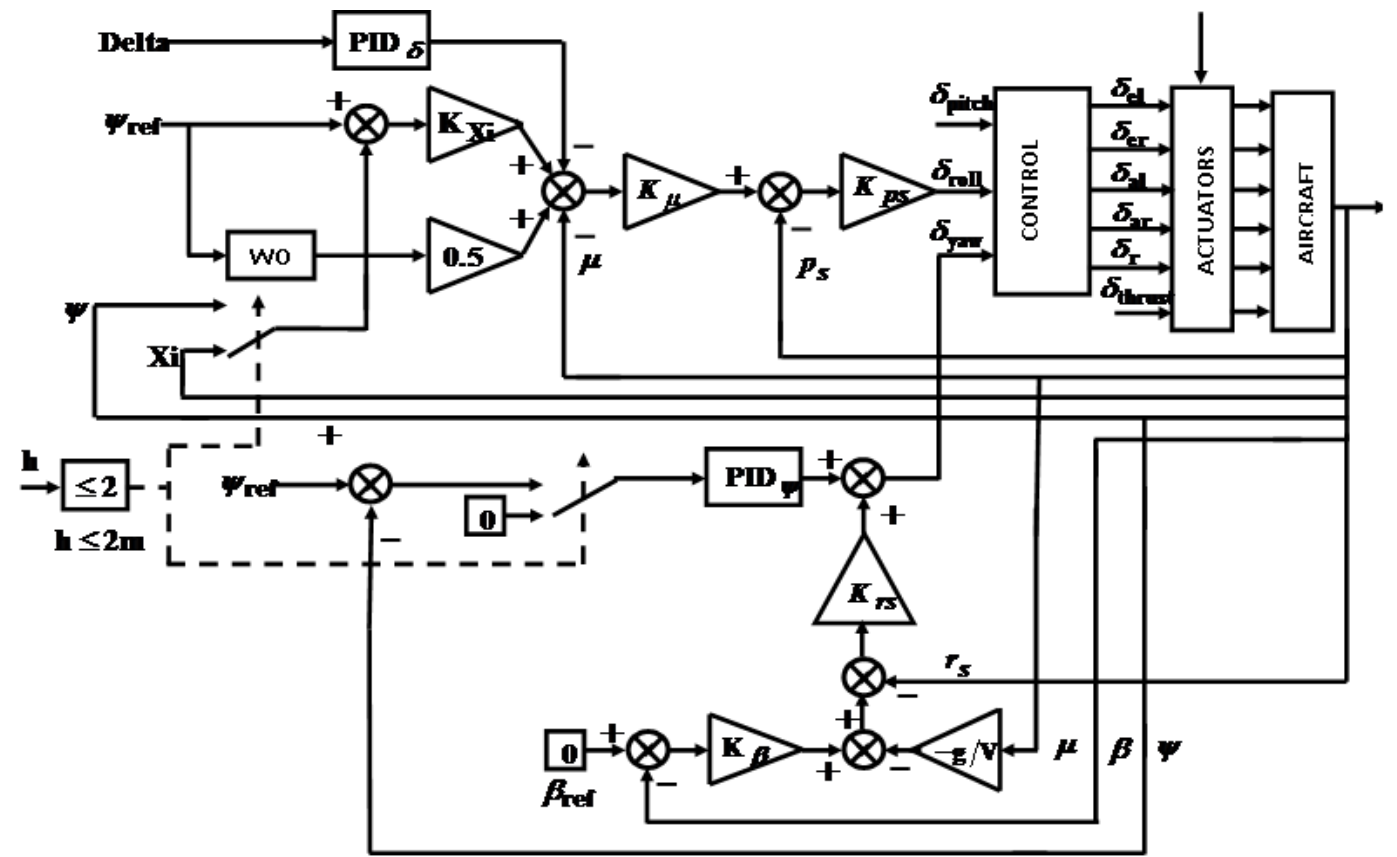

Fig. 6(ii) Lateral-Directional Axis FSFC
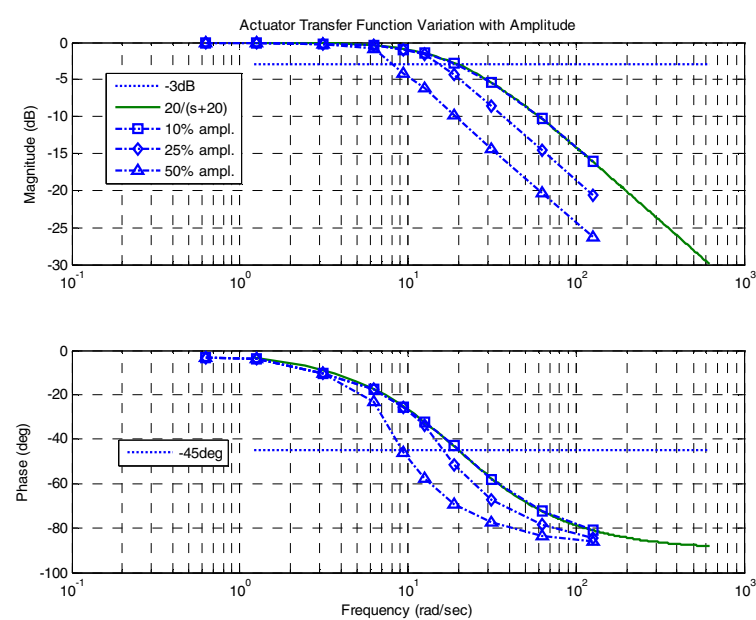

Fig. 7 Amplitude dependent frequency response of actuator

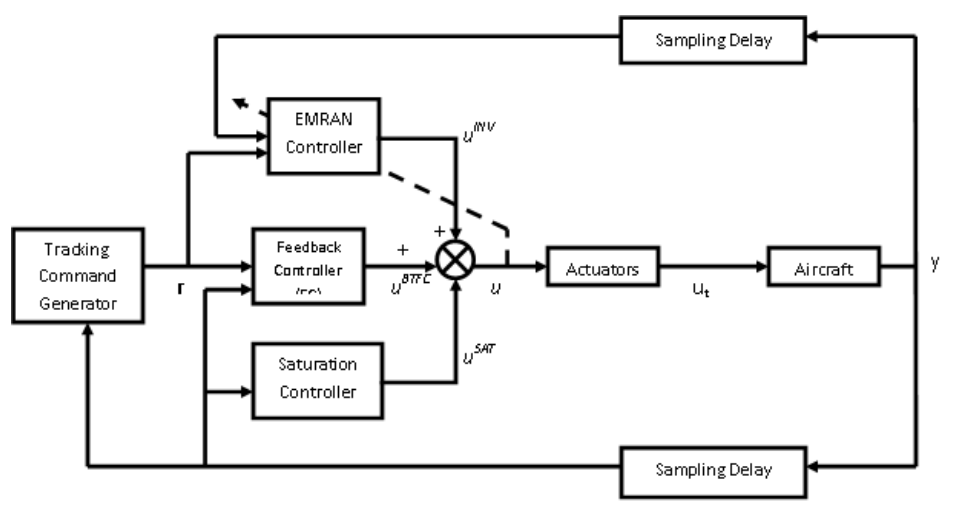

Fig. 8 Neural and SMC aided trajectory following controller

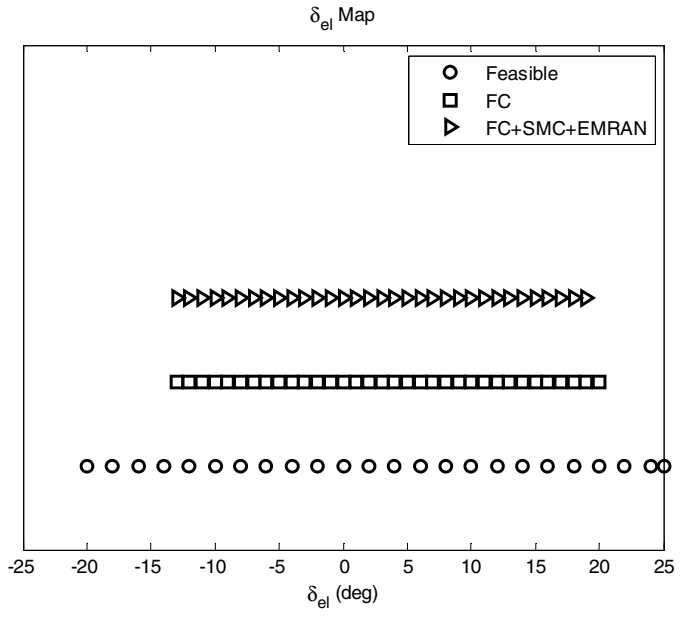

Fig. 9 Left elevator failure feasibility map

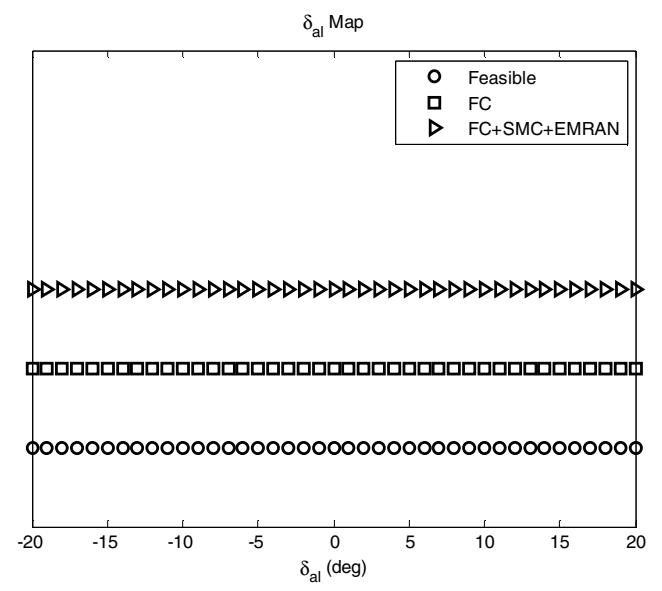

Fig. 10 Left aileron failure feasibility map 


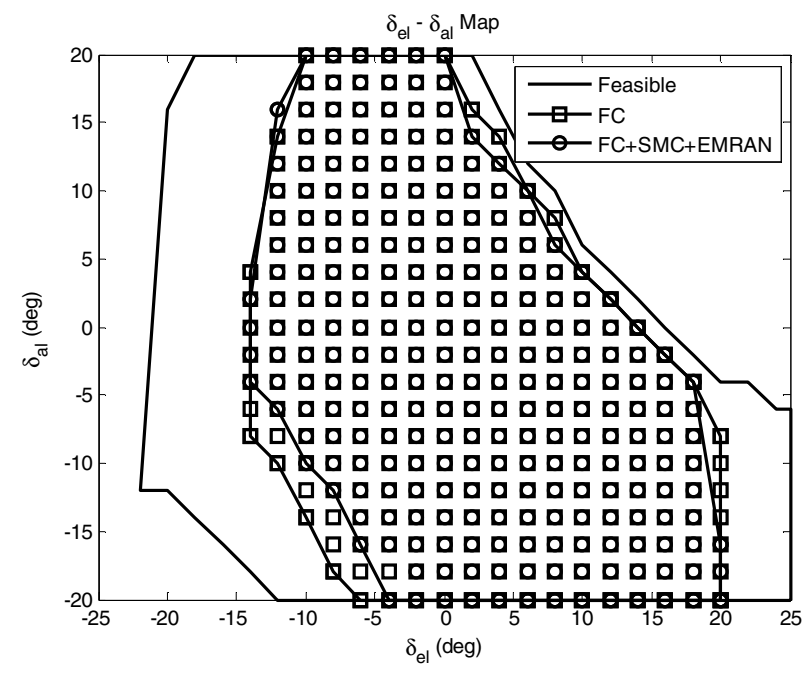

Fig. 11 Left elevator-left aileron failure feasibility map

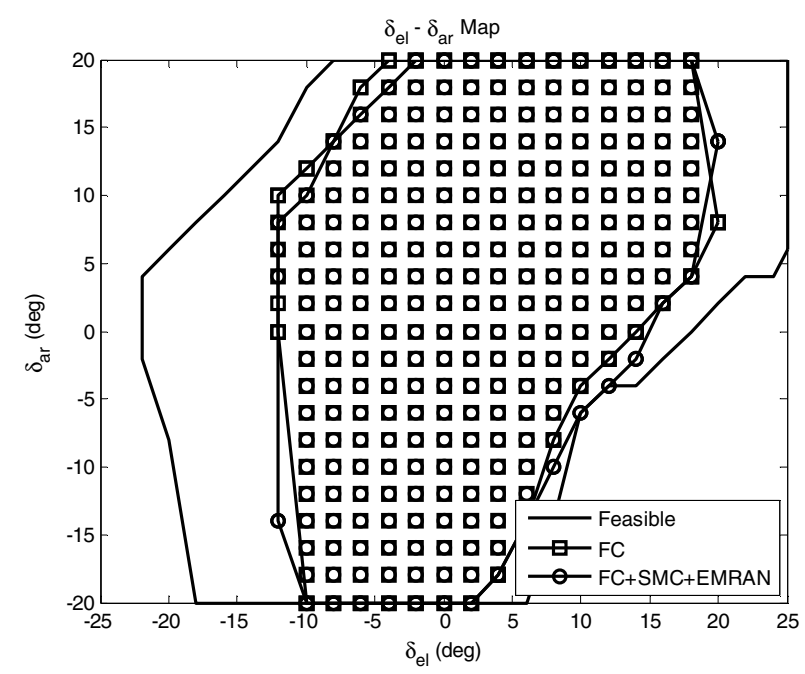

Fig. 12 Left elevator-right aileron failure feasibility map

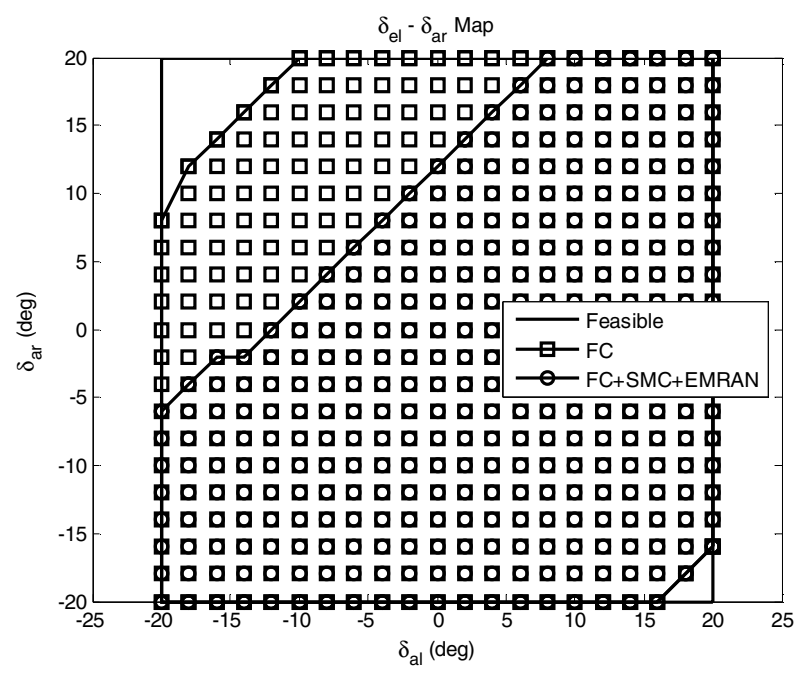

Fig. 13 Left aileron-right aileron failure feasibility map

$\delta_{\mathrm{r}}$ Map

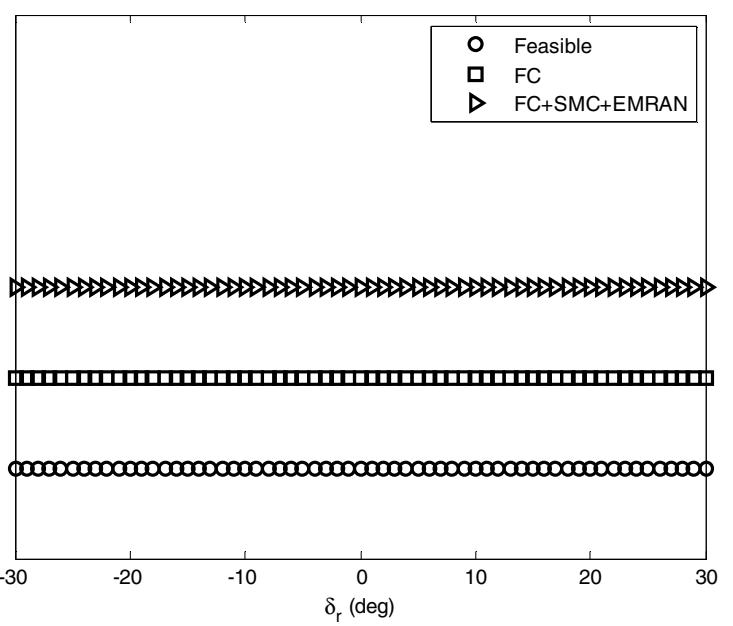

Fig. 14 Rudder failure feasibility map 